\title{
Grippe équine au Mali : résultats d'une enquête séroépidémiologique
}

\author{
S. Sidibé ${ }^{1}$ Z. Bocoum ${ }^{1}$ C.F. Simbé ${ }^{1}$ \\ K. Tounkara ${ }^{1}$ M.M. Bakkali ${ }^{2}$ M. Kané ${ }^{3}$
}

\section{Mots-clés}

Equidae - Influenzavirus équin Immunodiagnostic - Mali.

\begin{abstract}
Résumé
L'enquête a été réalisée dans les zones de Sayes, Fangasso, Tominian et San, situées dans la région de Ségou, dans la partie sahélienne du Mali, et a porté sur 384 sérums asins et équins. Parmi les 95 sérums positifs (24,73 p. 100), 92 I'ont été au test d'inhibition de l'hémagglutination et trois à la réaction de fixation du complément. Les taux d'infection aux sous-types 1 et 2 du virus de la grippe équine ont été établis dans les différentes zones visitées : 7,37 p. 100 pour le sous-type 1, 69,47 p. 100 pour le sous-type 2 et 23,16 p. 100 pour les cas d'infection mixte associant les sous-types 1 et 2 . L'enquête a aussi permis d'établir que le taux d'infection pour la maladie a varié selon I'espèce animale : il a été plus important chez les asins (35,02 p. 100).
\end{abstract}

\section{INTRODUCTION}

$\mathrm{Au}$ Mali, les équidés jouent un rôle socio-économique important. Leur effectif est estimé à plus de 737000 avec 85 p. 100 d'asins dont 16 p. 100 sont élevés dans la seule région de Ségou (8).

Dans plusieurs zones rurales du pays, les équidés sont régulièrement utilisés comme force de traction pour la culture attelée et le transport de personnes et de marchandises. Ils contribuent ainsi largement à l'augmentation des productions agricoles et à l'amélioration des conditions socio-économiques des populations rurales. Malgré leur rôle important, ils ne bénéficient pas de soins appropriés et subissent périodiquement de lourdes pertes causées par la grippe équine. En 1997, 7908 équidés ont contracté la grippe équine dans la région de Ségou, parmi lesquels 380 cas de mortalité ont été enregistrés (4,80 p. 100).

Il n'existe pas de programme national de lutte contre cette épizootie, l'action des services d'encadrement se limitant à la mise en œuvre d'un traitement symptomatique des infections bactériennes

1. Laboratoire central vétérinaire, Km 8, route de Koulikoro, BP 2295 , Bamako, Mali

Tél. : (223) 2243344 ; fax : (223) 2249809

E-mail : labovet@ datatech.toolnet.org

2. Laboratoire de produits biologiques et pharmaceutiques vétérinaires (Biopharma), Km 2, route de Casa, BP 4569, Akkari Rabat, Maroc

3. Direction nationale de l'Appui au monde rural, BP 2954, Bamako, Mali

Tél. : (223) 213914 secondaires. L'objet de la présente enquête a été d'étudier le taux d'infection des équidés par la grippe et la répartition des différents sous-types du virus dans les zones de Sayes, Fangasso, Tominian et San (figure 1) et de formuler des recommandations visant à contrôler la maladie.

\section{MATERIEL ET METHODES}

Les prélèvements ont été effectués dans des troupeaux d'équidés soupçonnés d'avoir été infectés par la grippe équine. Les sites de prélèvement ont été choisis en collaboration avec les agents des structures techniques d'encadrement et les agro-éleveurs. Les animaux étaient âgés de deux mois à 20 ans. La méthode de conduite des animaux était de type traditionnel. L'échantillon sanguin (5-10 ml) a été prélevé à la veine jugulaire dans des tubes Vacutainer stériles. Les sérums ont ensuite été répartis en aliquotes et conservés à $-20{ }^{\circ} \mathrm{C}$ avant de subir les examens sérologiques. Sur 384 échantillons de sérums récoltés, 177 ont été d'origine asine et 207 d'origine équine. Ils ont été testés au Laboratoire de produits biologiques et pharmaceutiques de Rabat, Maroc.

Pour l'analyse des sérums, deux techniques de diagnostic sérologique recommandées par l'Office international des épizooties (3) ont été utilisées : le test d'inhibition de l'hémagglutination et la réaction de fixation du complément. Les antigènes des sous-types 1 et 2 du virus et les antisérums de contrôle (positifs et négatifs) ont été fournis par le Laboratoire mondial de référence de l'Office international des épizooties pour le diagnostic de la grippe (Munich, Allemagne). 


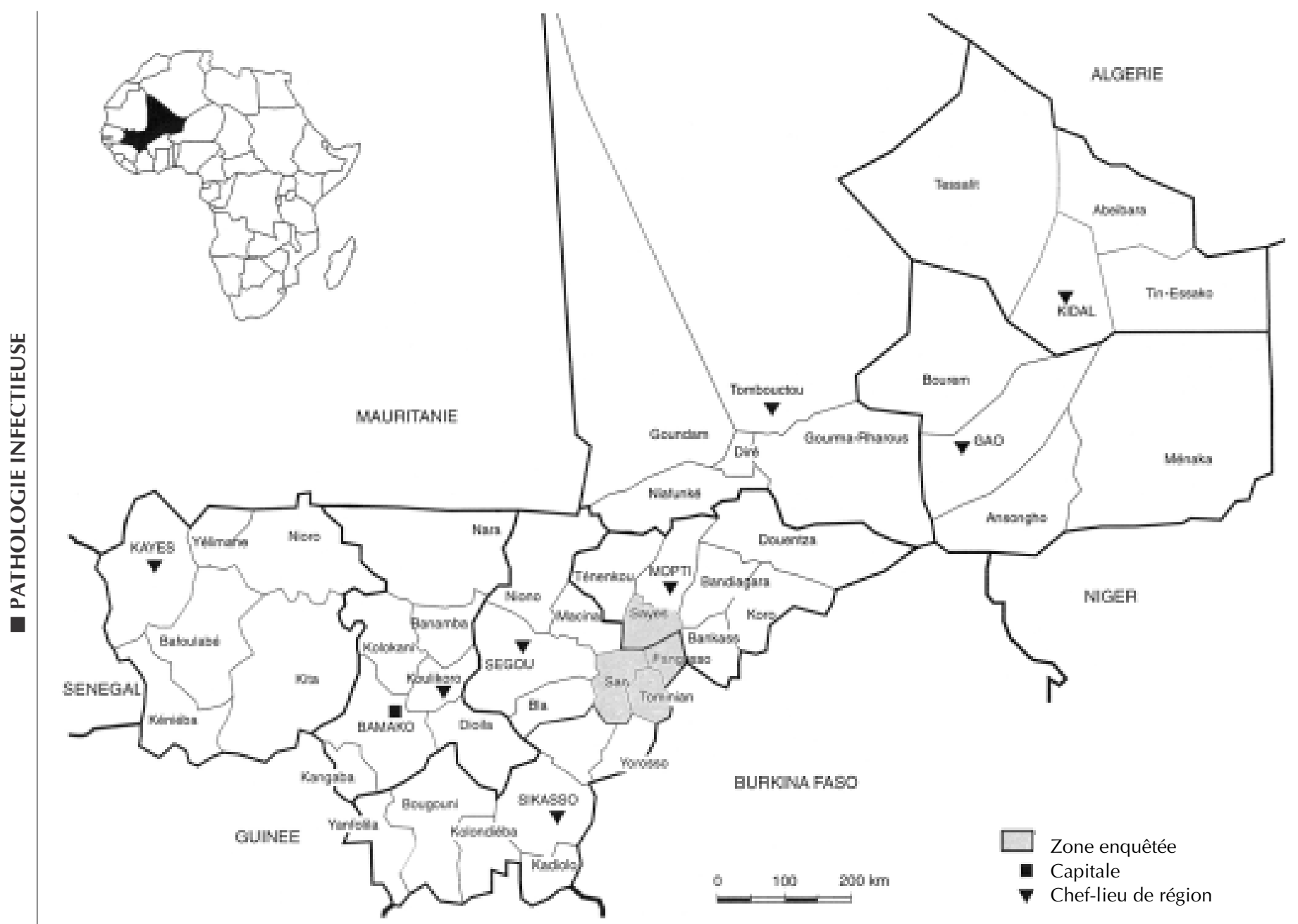

Figure 1 : carte du Mali montrant les zones de l'étude.

\section{RESULTATS}

Dans le cadre de l'enquête, 384 sérums asins et équins ont été testés, parmi lesquels 95 se sont avérés positifs $(24,73$ p. 100) : 92 au test d'inhibition de l'hémagglutination et trois à la réaction de fixation du complément.

Les résultats obtenus dans les différentes zones visitées (tableau I) ont montré que des anticorps dirigés contre le virus de la grippe équine ont été dépistés dans toutes les zones. Tous les échantillons de sérums positifs provenant des zones de Sayes, Fangasso et Tominian ont contenu des anticorps inhibant l'hémagglutination (96,84 p. 100). Ceux qui ont été positifs à la réaction de fixation du complément ont été dépistés seulement dans la zone de San.

La répartition des sous-types du virus a différé en fonction des localités visitées (tableau II). Ainsi, le sous-type 1 et les cas d'infections associant les sous-types 1 et 2 du virus de la grippe ont été identifiés dans toutes les zones couvertes par l'enquête, sauf à Tominian, où des anticorps vis-à-vis du seul sous-type 2 ont été mis en évidence. Ce dernier a prédominé dans toutes les zones visitées : il a été détecté dans 64,70 p. 100 des sérums testés positifs dans la zone de Sayes. A Fangasso et à San, les taux de prévalence des anticorps dirigés contre le sous-type 2 ont respectivement été de 52,94 et de 68,75 p. 100. La prévalence des anticorps vis-à-vis du sous-type 1 du virus a été plus importante à Sayes $(17,64$ p. 100) et l'a été beaucoup moins à San $(4,16$ p. 100). Le taux de prévalence a varié selon l'espèce animale (tableau III) : il a été plus élevé chez les asins $(35,02$ p. 100) et n'a atteint que 15,94 p. 100 chez les équins.
Les anticorps inhibant l'hémagglutination ont été identifiés chez 96,77 p. 100 des asins testés positifs, tandis que ceux fixant le complément ont été dépistés chez 3,22 p. 100 de ces animaux. Chez les équins, les taux de prévalence de ces deux types d'anticorps ont été respectivement de 96,96 et 3,03 p. 100.

\section{DISCUSSION}

Les échantillons testés ont été des sérums uniques. Deux techniques parallèles de diagnostic ont été utilisées : le test d'inhibition de l'hémagglutination et la réaction de fixation du complément. Le choix de la première méthode se justifie par le fait que les anticorps inhibant l'hémagglutination persistent dans le sérum plus longtemps et leur mise en évidence est la preuve de l'existence de cas d'infection ancienne. La deuxième technique, en revanche, permet de détecter les anticorps fixant le complément - preuve de l'existence d'une infection récente $(1,4,5,9,10)$.

Dans les zones de l'étude, le taux d'infection des équidés par la grippe a été de 24,73 p. 100. Les anticorps mis en évidence ont résulté d'une infection naturelle car les animaux porteurs de ces anticorps n'ont jamais été vaccinés contre la grippe. Ils ont été dirigés contre les sous-types 1 et 2 du virus de la maladie.

Cette enquête a permis d'établir que dans les localités de Sayes et Fangasso a circulé surtout le sous-type 1 du virus de la grippe. Il a été beaucoup moins répandu à San et absent à Tominian où seuls des anticorps dirigés contre le sous-type 2 ont été mis en évidence. 
Tableau I

Prévalence sérologique de la grippe équine dans les différentes zones visitées

\begin{tabular}{lccccccc}
$\begin{array}{l}\text { Localité } \\
\text { visitée }\end{array}$ & $\begin{array}{c}\text { Nb. sérums } \\
\text { testés }\end{array}$ & Nb. & \% & IH (nb.) & IH (\%) & Rfc (nb.) & Rfc $(\%)$ \\
\hline Sayes & & 17 & 24,28 & 17 & 100 & 0 & 0 \\
Fangasso & 70 & 17 & 23,28 & 17 & 100 & 0 & 0 \\
Tominian & 108 & 13 & 12,03 & 13 & 100 & 0 & 0 \\
San & 133 & 48 & 36,09 & 45 & 93,75 & 3,25 \\
Total & 384 & 95 & 24,73 & 92 & 96,84 & 3 & 3,15
\end{tabular}

IH : test de l'inhibition de l'hémagglutination

Rfc : réaction de fixation du complément

Tableau II

Répartition par localité visitée des sous-types du virus

\begin{tabular}{|c|c|c|c|c|c|c|c|}
\hline \multirow[t]{2}{*}{$\begin{array}{l}\text { Localité } \\
\text { visitée }\end{array}$} & \multirow[t]{2}{*}{$\begin{array}{l}\text { Nb. sérums } \\
\text { positifs }\end{array}$} & \multicolumn{2}{|c|}{$\begin{array}{l}\text { Sérums positifs } \\
\text { au sous-type } 1\end{array}$} & \multicolumn{2}{|c|}{$\begin{array}{l}\text { Sérums positifs } \\
\text { au sous-type } 2\end{array}$} & \multicolumn{2}{|c|}{$\begin{array}{c}\text { Sérums positifs } \\
\text { aux sous-types } 1 \text { et } 2 *\end{array}$} \\
\hline & & Nb. & $\%$ & Nb. & $\%$ & Nb. & $\%$ \\
\hline Sayes & 17 & 3 & 17,64 & 11 & 64,71 & 3 & 17,65 \\
\hline Fangasso & 17 & 2 & 11,76 & 9 & 52,94 & 6 & 35,29 \\
\hline Tominian & 13 & 0 & 0 & 13 & 100 & 0 & 0 \\
\hline San & 48 & 2 & 4,17 & 33 & 68,75 & 13 & 27,08 \\
\hline Total & 95 & 7 & 7,37 & 66 & 69,47 & 22 & 23,15 \\
\hline
\end{tabular}

* Infection mixte

\section{Tableau III}

Résultats par espèce animale des examens sérologiques

\begin{tabular}{lccccccc}
$\begin{array}{l}\text { Espèce } \\
\text { animale }\end{array}$ & $\begin{array}{c}\text { Nb. sérums } \\
\text { testés }\end{array}$ & Nb. & \% & IH (nb.) & IH (\%) & Rfc (nb.) & Rfc $(\%)$ \\
\hline Asine & & 62 & 35,02 & 60 & 96,77 & 2 & 3,22 \\
Equine & 177 & 33 & 15,94 & 32 & 96,96 & 1 & 3,03 \\
Total & 207 & 95 & 24,73 & 92 & 96,84 & 3 & 3,15
\end{tabular}

IH : test de l'inhibition de l'hémagglutination

$\mathrm{Rfc}$ : réaction de fixation du complément

Tous les virus isolés par Plateau et coll. (7) en France depuis 1979 appartiennent aussi au sous-type 2. En revanche, Ellouze (2) affirme que l'épizootie enregistrée en Tunisie en 1978-1979 a été causée par le sous-type $1 \mathrm{du}$ virus, avec une prévalence de 10 p. 100 pour le sous-type 2.

La présente étude a montré l'existence de cas d'infection mixte (infection d'un même sujet par les sous-types 1 et 2) dans toutes les zones de l'enquête, à l'exception de Tominian. Le taux élevé d'infection des asins par le virus de la grippe a montré que ces derniers ont été plus réceptifs à la maladie que les équins. Ainsi, le taux de prévalence de l'infection a été plus élevé à San, où l'effec- tif des asins a été plus important. Le taux le plus bas a été obtenu dans la zone de Tominian où l'espèce équine a prédominé.

En raison des disparités qui existaient au niveau du nombre des sérums collectés par tranche d'âge, les résultats relatifs aux taux d'infection n'ont pas permis de conclure qu'il existait une corrélation entre l'âge et le degré de réceptivité des animaux à la maladie (tableau IV). Une enquête réalisée par Bousseta et coll. (1) chez les équidés dans le Nord-Est tunisien a aussi confirmé qu'il existait une grande hétérogénéité de l'âge des animaux séropositifs envers la grippe. Nyaga et coll. (6) ont relevé des taux de séroprévalence nettement plus bas chez les sujets âgés de moins de deux mois. 
Tableau IV

Résultats par groupe d'âges des examens sérologiques

\begin{tabular}{lccc} 
Groupe d'âges & Nb. sérums testés & Nb. sérums positifs & \% sérums positifs \\
\hline $0-6$ mois & 3 & 0 & 0 \\
$1-5$ ans & 154 & 43 & 27,92 \\
$6-10$ ans & 196 & 47 & 23,97 \\
$11-15$ ans & 22 & 3 & 13,63 \\
$16-20$ ans & 9 & 2 & 22,22 \\
Total & 384 & 95 & 24,73
\end{tabular}

\section{CONCLUSION}

Le virus de la grippe équine (sous-types 1 et 2) circulait parmi les populations équine et asine des zones de Sayes, Fangasso, Tominian et San. L'étude a permis d'établir une prédominance nette des cas d'infection ancienne de grippe équine parmi les populations équine et asine étudiées (96,84 p. 100). Pour mieux contrôler la grippe équine en République du Mali, il est indispensable pour les services vétérinaires nationaux d'appliquer un programme de lutte basé sur la prophylaxie médicale par l'usage d'un vaccin à base des sous-types 1 et 2 du virus de la maladie.

\section{Remerciements}

Les auteurs adressent leurs remerciements à la direction du programme de Diversification des revenus en zones non-cotonnières (San) pour le financement de l'enquête et au directeur général de la société de Produits biologiques et pharmaceutiques vétérinaires (Biopharma) d'Akkari Rabat, Maroc, pour sa franche collaboration.

\section{BIBLIOGRAPHIE}

1. BOUSSETA M., CHABCHOUB A., GHRAM A., JOMAA I., GHORBEL A., AOUINA T., BEN AMOR H., 1994. Enquête séroépidémiologique sur la grippe équine et I'anémie infectieuse des équidés dans le Nord-Est tunisien. Revue Elev. Méd.vét. Pays trop., 47 : 277-281.

\section{Summary}

Sidibé S., Bocoum Z., Simbé C.F., Tounkara K., Bakkali M.M., Kané M. Equine Influenza in Mali: Results of a Seroepidemiological Survey

The survey was carried out in Sayes, Fangasso, Tominian and San areas, located in the region of Segou in the Sahelian zone of Mali, and concerned 384 equine and donkey sera. Of the 95 positive sera $(24.73 \%), 92$ were positive by the hemagglutination inhibition test and three by the complement fixation test. The infection rates by subtypes 1 and 2 of the equine influenzavirus found in the various visited areas were as follows: $7.37 \%$ by subtype $1,69.47 \%$ by subtype 2 , and $23.16 \%$ by subtypes 1 and 2 (mixed infection). The survey also showed that the infection rate of the disease varied according to the animal species: it was higher in donkeys $(35.02 \%)$

Key words: Equidae - Equine influenzavirus - Immunodiagnosis - Mali.
2. ELLOUZE M.R., 1980. Contribution à l'étude épidémiologique de I'anémie infectieuse, de l'artérite à virus, de la rhinopneumonie et de la grippe équine en Tunisie. Thèse Doct. vét., Sidi-Thabet, Tunisie.

3. Equine influenza. Manual of standards for diagnostic tests and vaccines, 1996. Paris, France, OIE, p. 409-419.

4. FONTAINE M., MORAILLON A., 1980. Considération sur l'épizootie de grippe 1978-1979 en France. Recl Méd. vét., 156 : 139-145.

5. LE MINOR L., VERON M., 1982. Bactériologie médicale. Paris, France, Flammarion Médecine-Sciences, p. 114-115.

6. NYAGA P.N., WIGGINS A.D., PRIESTER W.A., 1980. Epidemiology of equine influenza, risk by age, breed and sex. Comp. Immun. Microbiol. infect. Dis., 3: 67-73.

7. Plateau E., CRuCiere C., JACQuet A., CHEyrouX M., 1984. Mise au point et recherche en cours sur l'évolution épidémiologique et antigénique de la grippe équine en France. Bull. mens. Soc. Vét. Prat. Fr., $68: 6-10$.

8. Recueil des statistiques du secteur rural malien, 1998. Bamako, Mali, ministère du Développement rural et de l'eau, Cellule de planification et de statistique, $88 \mathrm{p}$.

9. TIZARD I., 1977. An introduction to veterinary immunology. Philadelphia, PA, USA, W.B. Saunders, p. 141-142.

10. ZIENTARA S., PLATEAU E., 1992. Vaccins et vaccinations chez le cheval. Point vét., 24 : 601-610.

Reçu le 01.08.2001, accepté le 23.01.2003

\section{Resumen}

Sidibé S., Bocoum Z., Simbé C.F., Tounkara K., Bakkali M.M., Kané M. Gripe equina en Malí: resultados de una encuesta sero-epidemiológica

La encuesta se realizó en las zonas de Sayes, Fangazo, Tominian y San, situadas en la región de Ségou, en la parte sahariana de Malí y sobre 384 sueros de asnos y equinos. Entre los 95 sueros positivos $(24,73 \%), 92$ lo fueron mediante el test de inhibición de la hemoaglutinación y tres mediante la reacción de fijación de complemento. En las diferentes zonas visitadas, se detectaron tasas de infección de los sub-tipos 1 y 2 del virus de la gripe equina: $7,37 \%$ para el sub-tipo 1, 69,47\% para el sub-tipo 2 y $23,16 \%$ para los casos de infección mixta asociando los sub-tipos 1 y 2 . La encuesta permitió también establecer que la tasa de infección para la enfermedad varió según la especie animal: fue más importante en los asnos $(35,02 \%)$.

Palabras clave: Equidae - Virus de la influenza equina Inmunodiagnóstico - Mali. 